\title{
Thermal Conductivity of Concentrated Colloids in Different States
}

\author{
Natallia Shalkevich, ${ }^{\dagger}$ Andrey Shalkevich,,${ }^{\dagger}$ and Thomas Bürgi ${ }^{*}, \dagger, \$$ \\ Institut de Physique, Laboratoire de chimie physique des surfaces, Université de Neuchâtel, Rue Emile-Argand 11, \\ 2009- Neuchâtel, Switzerland, Adolphe Merkle Institute and Fribourg Center for Nanomaterials, \\ University of Fribourg, Route de l'ancienne Papeterie CP 209, CH-1723 Marly, Switzerland, and \\ Physikalisch-Chemisches Institut, Ruprecht-Karls-Universität Heidelberg, Im Neuenheimer Feld 253, \\ 69120 Heidelberg, Germany
}

\section{Introduction}

Heat transfer is very important in many industrial and technical processes. Various fluids are commonly used as heat conductors. Nevertheless, they have too low thermal conductivity for certain applications and various additives are used to improve the situation. The most common way is the addition of dispersed solid particles to the fluid. The thermal conductivity of the suspension with millimeter- or micrometer-sized welldispersed particles can be estimated within effective medium theory by the classical Maxwell-Garnett model. ${ }^{1-3}$ This model is based on different aspects such as spherical solid particles, volume fraction, and thermal conductivity of fluid and solid materials and generally works well for a low thermal conductivity ratio $(<10)$ between the solid and the fluid. The MaxwellGarnett model was further extended by several investigators who included the effect of shape, ${ }^{4,5}$ particle interactions, ${ }^{2,6-11}$ and particle distribution. ${ }^{12}$ Meanwhile, there are many experimental data which have shown that composite fluids with nanoparticles, also known as nanofluids, have much higher thermal conductivity as predicted by effective medium theory. ${ }^{13}$

A number of publications ${ }^{2,14-16}$ on thermal properties of nanofluids show very large enhancements in the thermal conductivity of nanofluids. The most extreme results were obtained for different allotropes of carbon. For example, enormous enhancements of thermal conductivity by $160 \%$ and by $70 \%$ were observed for a suspension containing $1 \%$ of MWCNTs in oil ${ }^{16}$ and for $1 \%$ ultradispersed diamond in ethylene glycol, respectively. ${ }^{2}$ It should, however, be noted that even with these drastic enhancements the thermal conductivity of the complex fluids is lower than the one of pure water $(0.609$ $\mathrm{W} / \mathrm{mK}$ ) due to the low thermal conductivity of the base fluids (oil, $0.145 \mathrm{~W} / \mathrm{mK}$; ethylene glycol, $0.258 \mathrm{~W} / \mathrm{mK}$ ). Currently, there are several routes for explaining the mechanism responsible for unusually high thermal transport properties of nanof-

\footnotetext{
* Corresponding author. E-mail:buergi@uni-heidelberg.de.

${ }^{\dagger}$ Institut de Physique.

* University of Fribourg.

$\S$ Ruprecht-Karls-Universität Heidelberg.
}

luids, such as effects of liquid layering at solid interface, ${ }^{17-19}$ particle Brownian motion, ${ }^{20-23}$ and particle clustering. ${ }^{2,6-11}$

One of the major mechanisms of thermal conductivity enhancement is the layering of the solvent molecules on the particle surface. Liquid molecules form an ordered solid-like layer around the particle that would have a thermal conductivity higher than the bulk liquid medium (Keblinski et al. ${ }^{17,24}$ ) and could give a path for rapid heat transfer between a solid particle and the base fluid. Liquid layering theory was shown to be promising but it uses as adjustable parameter the thickness of the liquid layer. Furthermore, experimental verification is not trivial at all.

Brownian motion of nanoparticles can influence the thermal conductivity by two independent scenarios. The first of them assumes that the particles with higher kinetic energy absorb the heat from the fluid surrounding them, migrate to another region, and release the thermal energy to the surrounding liquid, thereby enhancing the thermal transport. ${ }^{20,21}$ But Evans et al. have recently demonstrated ${ }^{25}$ that such a contribution of Brownian motion of nanoparticles to heat transfer is negligible in comparison with much faster thermal diffusion through the base fluid. A second scenario ${ }^{23}$ is based on the hydrodynamic effect of the moving particles, which results in locally ordered microconvection of the base fluid around each nanoparticle. Recent molecular dynamics simulations made by Evans et al. ${ }^{26}$ revealed that the hydrodynamic Brownian motion mechanism has only a minor effect on the effective thermal conductivity for a relatively high particle volume fraction (ca. 3.3 vol \%).

Depending on the chemistry of the system particle aggregation can take place. At constant volume fraction the probability of aggregation increases with decreasing particle size, because the average interparticle distance decreases, making the attractive van der Waals force more important. ${ }^{22}$ Large and sparse clusters can even decrease thermal conductivity ${ }^{8}$ while chain-like clusters pass through the whole volume and significantly enhance heat transfer, ${ }^{6}$ which was recently confirmed by simulations. ${ }^{7,8,27}$ Keblinski and Prasher attribute the thermal conductivity enhancement mainly to the ability of the heat to move rapidly along the backbone of the aggregate named also as a high- 
conductivity percolation path. Some experimental observations confirmed the role of the clustering for the increase of thermal conductivity of nanofluids. ${ }^{8-10}$ Philip et al. ${ }^{10}$ demonstrated $216 \%$ enhancement of thermal conductivity for a magnetite nanofluid in a magnetic field $(101 \mathrm{G})$ where $\mathrm{Fe}_{3} \mathrm{O}_{4}$ nanoparticles form a chain-like structure.

The present work investigates the effect of the particles motion and arrangement on the thermal conductivity of colloidal suspensions. We are going to compare thermal properties of a system of freely moving particles (fluid) with suspensions where particles are "frozen", i.e., restricted in translational motion (gel and glass). Moreover, we are going to test the influence of the particle-particle contact on the heat transfer by comparison of a system with interconnected particles (gel) and one with fully separated particles (glass). For this purpose, we used two kinds of nanoparticles with significantly different thermal conductivity of their bulk solid. Scattering methods as well as rheology were applied to determine their degree of motion and to observe the overall structure of these suspensions.

\section{Experimental Section}

Materials. Ludox-grade TMA and TM colloidal silica (22 $\mathrm{nm}$ in diameter) suspensions in deionized water (34 wt \% for TMA and 50 wt \% for TM) were purchased from AldrichSigma. Colloidal alumina (30 $\mathrm{nm}$ in diameter) suspension in water (20 wt \%) was purchased from Nanostructured and Amorphous Materials Inc. All reagents were used as received. Dowex DM80 was purchased from Aldrich-Sigma. Milli-Q (Millipore) water with a resistivity of $18.2 \mathrm{M} \Omega$ was employed throughout.

Instrumentation. The rheological behavior of dispersions was studied with an MCR 300 rheometer (Paar Physica) in temperature-controlled narrow-gap Mooney-Ewart geometry. ${ }^{28}$ All tests were done at $25{ }^{\circ} \mathrm{C}$.

Dynamic light scattering (DLS) was performed with an ALV5000 spectrophotometer equipped with an argon-laser (Coherent, model Innova $300, \lambda=488 \mathrm{~nm}$ ), a digital autocorrelator (ALV), and a variable angle detection system. Measurements were made at a fixed scattering angle of $90^{\circ}$ and a temperature of $25.0 \pm$ $0.1^{\circ} \mathrm{C}$. The individual correlation functions were analyzed using a second-order cumulant fit. Gel and glass samples were studied with DLS measurements by using 3D cross correlation setup from LS Instruments GmbH, Fribourg, Switzerland. This instrument allows the suppression of multiple scattering, as described in detail elsewhere. ${ }^{29}$

Transmission electron micrographs (TEM) were obtained with a Philips C 200 microscope in bright field mode at a voltage of $200 \mathrm{kV}$. The samples for TEM study were prepared by casting a few drops of the colloid onto carbon-coated copper grids (300 mesh) and used after solvent evaporation in air.

The KD2 Themal Properties Analyzer from Decagon was used for all measurements of the thermal conductivity and it works on a similar principle to the transient hot wire method. It calculates the thermal conductivity of samples by measuring the dissipation of energy from a line heat source. ${ }^{30}$ Each measurement takes a total of $90 \mathrm{~s}$, leaving the temperature to stabilize during the first $30 \mathrm{~s}$, heating for the second $20 \mathrm{~s}$, and determining the rate of cooling in the final $30 \mathrm{~s}$, with final values of thermal conductivity being quoted to an accuracy of $5 \%$. The KD2 works on the assumption that the probe is an infinitely long heat source and that the material (fluid or solid) being measured is homogeneous and isotropic and of a uniform initial temperature. The relationship between $\Delta T$ and $\ln (t)$ can be shown as in eq $1:^{30}$

$$
T-T_{0} \cong \frac{q}{4 \pi \lambda_{\mathrm{h}}}\left(\ln (t)-\gamma-\ln \left(\frac{r^{2}}{4 \kappa}\right)\right)
$$

where $T=$ temperature $(\mathrm{K}), T_{0}=$ initial temperature $(\mathrm{K}), q=$ heat produced per unit length per unit time $\left(\mathrm{W} \mathrm{m}^{-1}\right), \lambda_{\mathrm{h}}=$ thermal conductivity of the medium $\left(\mathrm{W} \mathrm{m}^{-1} \mathrm{C}^{-1}\right), t=$ time (s), $\gamma=$ Euler's constant, $r=$ radial distance $(\mathrm{m})$, and $\kappa=$ thermal diffusivity $\left(\mathrm{m}^{2} \mathrm{~s}^{-1}\right)$.

By plotting $\Delta T$ against $\ln (t)$, the thermal conductivity is then simply calculated from the gradient of the slope, $m$, which is equal to $q / 4 \pi \lambda_{\mathrm{h}}$.

Preparation of Colloids. Formation of Gel State. One of the common routes of gel formation is a collapse of the electrical double layer (EDL) on the particle surface by adding of electrolyte. Obviously, the Coulomb repulsion is now so strongly screened that interparticle van der Waals attractions become dominant and the particles become unstable. Destabilized particles stick together and form a percolated network. ${ }^{31}$ Macroscopic properties of gel are determined by the network parameters (density, bond strength, etc.), which depend on the composition and the concentration of added electrolyte. ${ }^{32}$ Sodium (or potassium) chloride is normally used as destabilizing agent to prepare a gelled colloid. At a concentration of $0.2 \mathrm{M}$ this salt can reduce the EDL thickness to $1 \mathrm{~nm}^{32}$ and stabilizing repulsion forces become negligible.

The colloids of $\mathrm{SiO}_{2}\left(\mathrm{Al}_{2} \mathrm{O}_{3}\right)$ nanoparticles in water were prepared as follows. Several drops of 7-8 $\mathrm{M}$ solution of $\mathrm{NaCl}$ were added to $50 \mathrm{~mL}$ of silica (alumina) aqueous suspension up to $0.2 \mathrm{M}$ final concentration of $\mathrm{NaCl}$. This suspension was stirred 5 min and kept for several days to equilibrate and form the network structure. Samples for dynamic light scattering measurements were prepared directly in the measuring cell to avoid structure distortion.

Formation of Glass State. To prepare a colloid in glassy state, one has to increase the EDL of particles that can be realized by addition of exchange resin. The latter removes the counterions from the colloid, thus increasing the electrical double layer. Extending the EDL, the interparticle electrostatic repulsion forces and the effective volume of EDL-particle increase simultaneously. At some point the moving of the particles becomes restricted at the finite threshold and the cage effect is observed when the particle motion is limited in space bound to neighbor particles. In this case, the particles are well separated one from another; i.e., there is no direct contact between particles. ${ }^{33-36}$ The absence of particle translational motion eliminates the solvent convection. Furthermore, the majority of the solvent molecules are immobilized in the EDL, and therefore, traditional heat transfer due to the diffusion of solvent is significantly reduced.

To form the glassy state, an initial nanoparticle colloid of required concentration was mixed with ion-exchange resin (Dowex). An amount of resin corresponding to one-fifth of the total volume was used. The mixture was kept at least for 1 week to remove excess ions. Once the resin was mixed with the suspension, we periodically ( 2 times per day) agitated the sample tube. Afterward, the thickened dispersion was filtered through $5 \mu \mathrm{m}$ pore size Millipore filters to remove all ion-exchanged beads.

\section{Results}

We have studied suspensions of silica and alumina nanoparticles with diameters of 22 and $30 \mathrm{~nm}$, respectively. Their sizes were confirmed by transmission electron microscopy (TEM) 
(a)

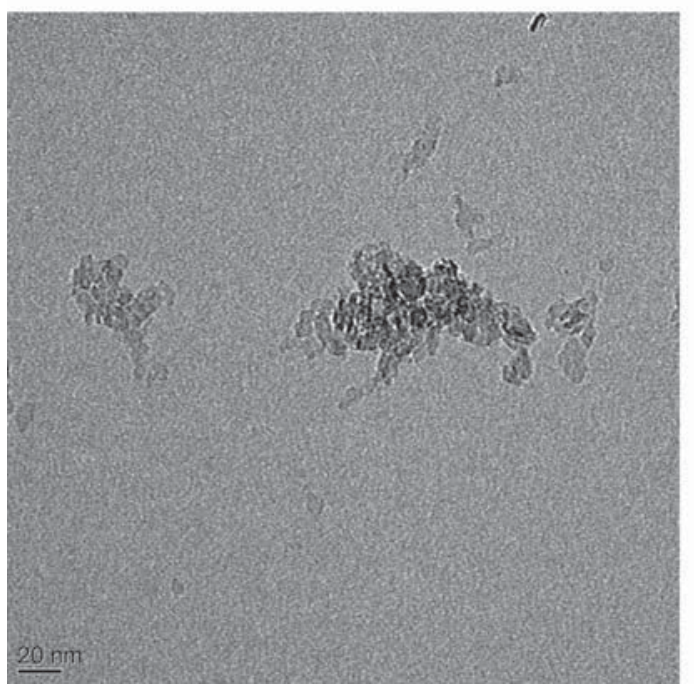

(b)

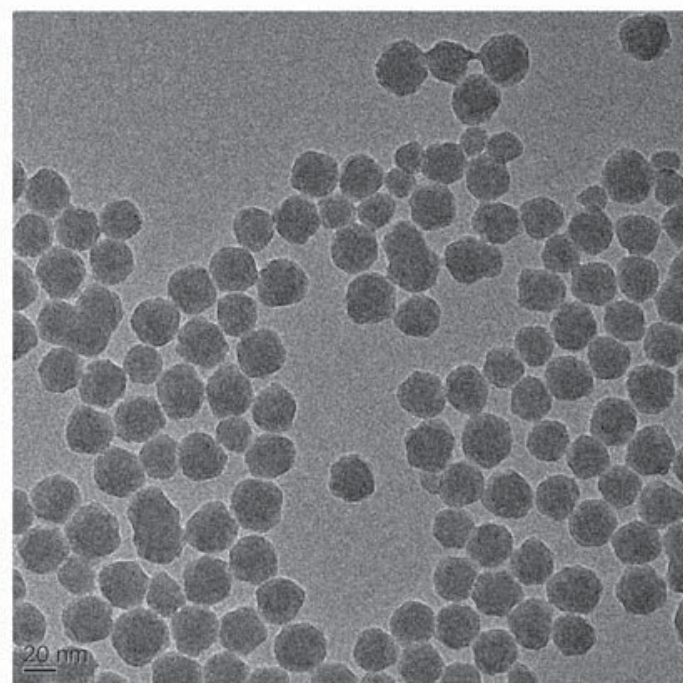

Figure 1. Transmission electron microscopy graphs of alumina (a) and silica (b) nanoparticles.
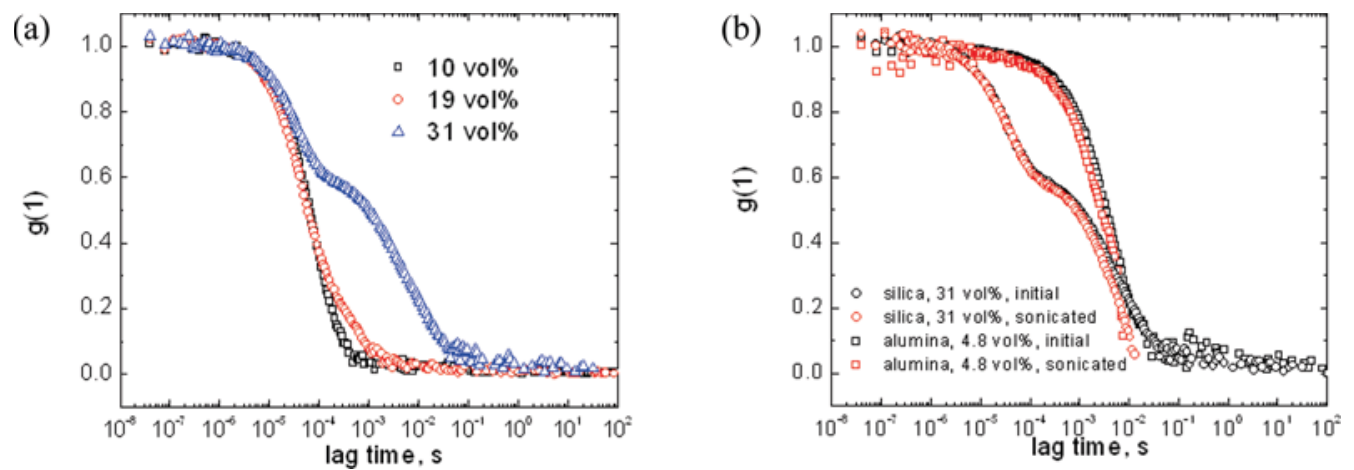

Figure 2. Measured intensity correlation functions as a function of decay time for (a) silica suspensions at different concentrations and (b) a silica and alumina suspension in initial states and after sonication.

imaging, as shown in Figure 1. While silica particles have a spherical shape and diameter of $22 \mathrm{~nm}$, as reported by the producer, the alumina particles are irregularly shaped with considerable polydispersity $(10-30 \mathrm{~nm})$ and probably porous morphology. To connect those observations with the structure in the fluid samples, we performed DLS measurement on the most diluted samples and a subsequent cumulant fit of the correlation functions. The silica suspension exhibits low polydispersity and a mean hydrodynamic radius of $16 \mathrm{~nm}$ in the concentration range from 0.2 to 19 vol \% (Figure 2a). The increase of diameter of small silica particles determined by DLS as compared to TEM is well-known and normally attributed to the roughness and/or hairing of the silica surface. ${ }^{37,38}$ The alumina suspension demonstrates distinctly different behavior. Dynamic light scattering not only reveals the signal from individual (and rather polydisperse) particles but also indicates the existence of very polydisperse clusters with mean hydrodynamic radius of around $100 \mathrm{~nm}$ (Figure 2b).

The influence of the cluster formation on the thermal conductivity of nanofluids has been reported in many studies. Therefore, we have tested the effect of particle concentration on the presence of particle clusters (aggregates), and the results for three concentrations of silica are shown in Figure 2a. While samples with 10 and 19 vol \% of silica have almost the same behavior and no aggregates, the suspension with maximum silica concentration demonstrates a clear sign of aggregates. To test the stability of the clusters, we have performed dynamic light scattering measurements of initial suspensions and of suspen- sions after treatment with a Branson sonifier 250 over 15 min (setting duty-cycle 50\%, output control 5). As shown in Figure $2 \mathrm{~b}$, both the silica and alumina suspensions do not exhibit significant change after ultrasonic treatment due to either the high stability of the aggregates or their fast reassembly due to the high concentration of the particles. Nevertheless, a small amount of clusters (which are intermediate state between individual particles and gel network) in the fluid is expected to have a much smaller influence on the thermal conductivity of the fluid than a fully established percolated network.

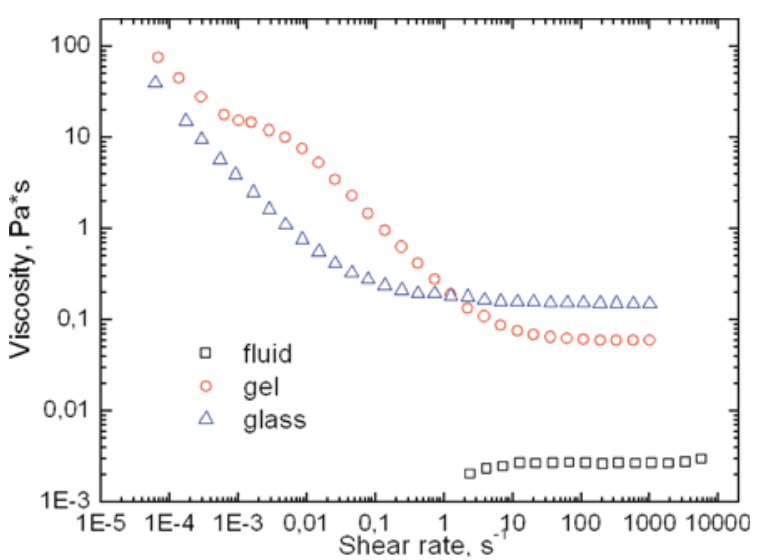

Figure 3. Viscosity as a function of shear rate for silica suspensions of $19 \mathrm{vol} \%$ in fluid, gel, and glass states. 

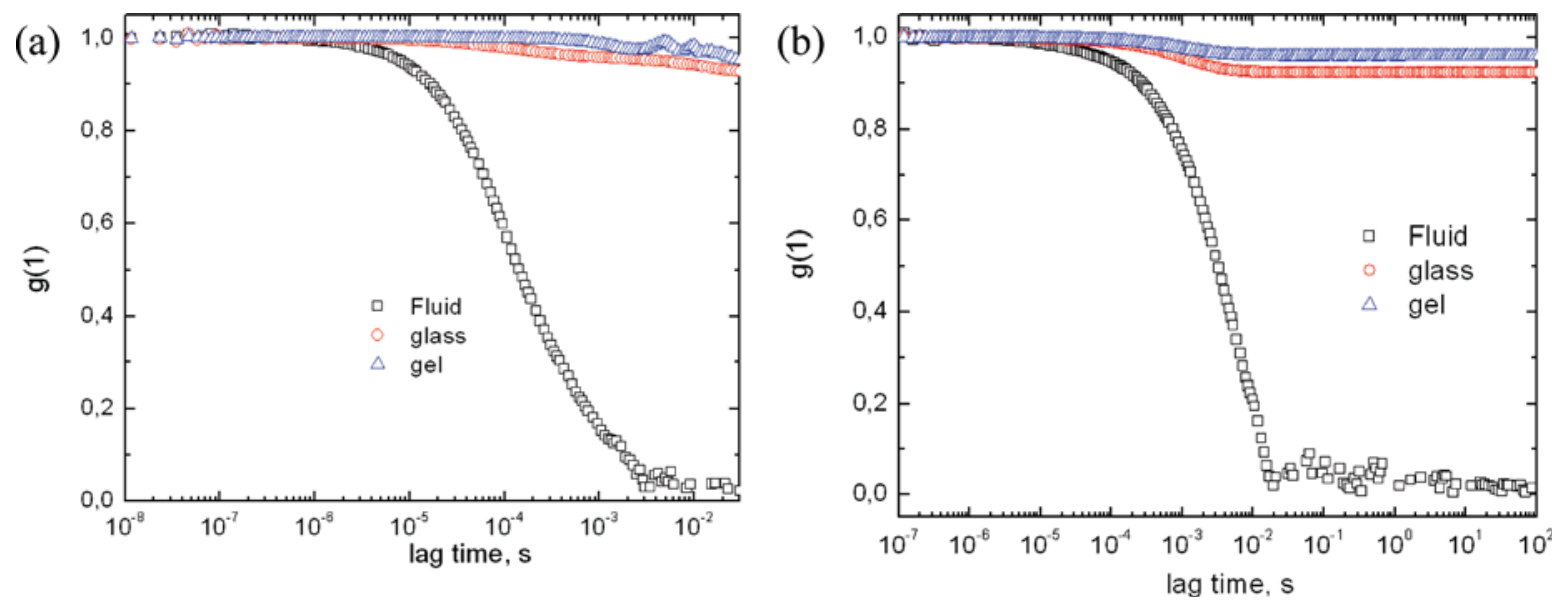

Figure 4. Measured intensity correlation functions as a function of decay time for different states of (a) a 19 vol \% silica suspension and (b) a 4.8 vol \% alumina suspension.

To test macroscopic structures of different colloid states and their influence on the heat transfer, we performed rheological measurements of silica suspensions at 19 vol \%. We have used the same batch of initial silica nanoparticle suspension (TMA) for the preparation of all colloidal states at identical concentrations, and therefore, the only difference is particle interactions and structure. Results are shown in Figure 3. The fluid-like sample exhibits typical behavior of Newtonian fluid with viscosity independent of the shear rate. Due to low viscosity values, we were able to detect significant shear stress only at high shear rates. When shear rates decrease, the viscosity drops and becomes undetectable. For structured colloidal dispersions (gel and glass) subjected to steady shear, we observed that we have a high-shear Newtonian plateau at accessible shear rates. At low shear values the glassy and gelled samples exhibit the shear-thinning behavior with slope of -1 over more than two decades in shear rates, which is similar to the behavior observed previously in colloidal suspensions undergoing a liquid-solid transition. ${ }^{28} \mathrm{~A}$ weak plateau for the gel-like sample at low shear rates can be the result of complex structural properties of the sample, such as network subunits, which require additional shear stress to break.

To study the local dynamics of the particles in different colloidal states, we have again performed dynamic light scattering. Results are presented in Figure 4. We observed almost the same behavior for the gel and glass states where we see clearly nonergodic behavior as a result of structure formation. While the fluid sample demonstrates classical Brownian motion of particles, the nonergodic samples have only traces of selfdiffusion and very slow collective diffusion motion. The silica and alumina suspensions exhibit the same peculiarities and indeed can be compared for the heat transfer.

Experimental data for the relative thermal conductivity of silica and alumina in different colloidal states are summarized in the Figure 5. Milli-Q water was used as the reference base fluid for the fluid sample. For the gel and glass systems the water base fluid was additionally modified to gain accurate relative thermal conductivity values. We have used a $0.2 \mathrm{M}$ solution of sodium chloride for the gelled colloids, and for glassy samples the water was treated with Dowex exchange resin. The thermal conductivity of the reference base fluid was measured independently at the same temperature as the colloids. The measurement temperature was kept at $22 \pm 1{ }^{\circ} \mathrm{C}$.

The thermal conductivity of silica suspensions in fluid and gel states increases almost linearly with concentration, showing

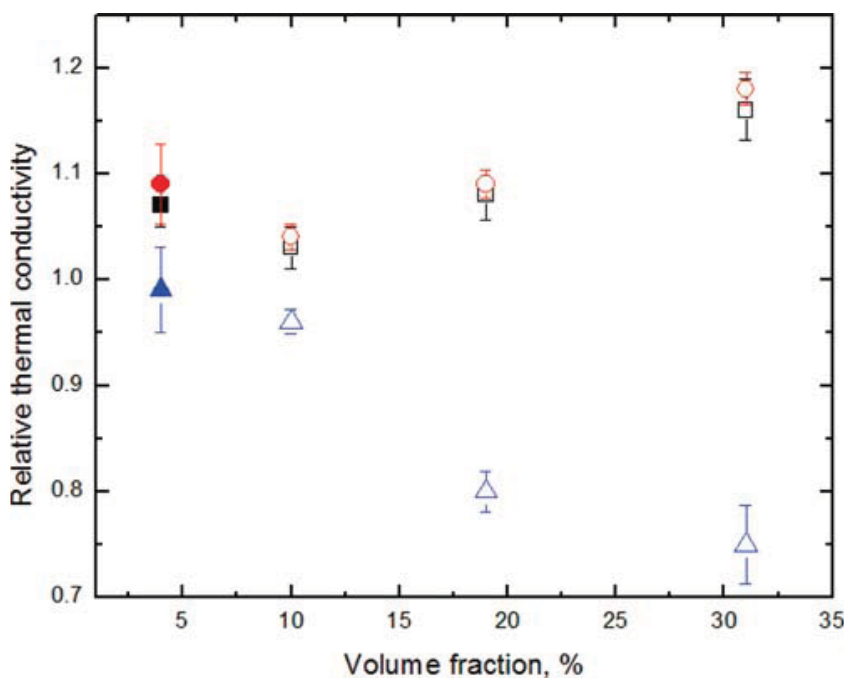

Figure 5. Thermal conductivity relative to the base fluid of several suspensions: $(\square)$ silica fluid; $(\bigcirc)$ silica gel; $(\Delta)$ silica glass; $(\mathbb{\square})$ alumina

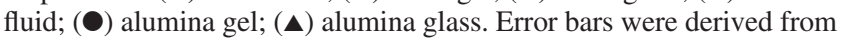
at least 3 series of measurements (different points in the sample) with at least 10 individual measurements per series.

a maximum thermal conductivity of $0.706 \mathrm{~W} \mathrm{~K}^{-1} \mathrm{~m}^{-1}$ or $18 \%$ enhancement for the gel state at a volume fraction of $31 \%$. Similar behavior of silica colloids in the fluid state was observed by other groups. ${ }^{39}$ In contrast, the thermal conductivity of the glass state strongly decreases with increasing volume fraction and drops to $0.449 \mathrm{~W} \mathrm{~K}^{-1} \mathrm{~m}^{-1}$ or $-25 \%$ for the glass with a silica concentration of $31 \mathrm{vol} \%$. Alumina suspensions at the lower volume fraction of $4.8 \mathrm{vol} \%$ demonstrate higher thermal conductivity enhancement than silica suspensions at $10 \mathrm{vol} \%$ in fluid and gel states as well as a larger improvement for the gelled sample with respect to the fluid one. The glassy alumina sample does not exhibit any significant thermal conductivity enhancement.

\section{Discussion}

The main speculations on the enhanced thermal conductivity of nanofluids relate to Brownian motion ${ }^{20,21,23}$ and to the nanoparticles organization in the fluid. ${ }^{6-9}$ Thus, the Brownian motion of the particles is drastically changed if particles aggregate and form clusters and/or networks. Aggregation may influence as well the liquid layering effect due to decreasing accessible particle surface for the solvent molecules. We have 
observed that the gelled samples that form networks of directly contacting nanoparticles demonstrate slightly better thermal conductivity enhancement than the fluid state. This indicates that Brownian motion does not play the key role in heat transfer of concentrated suspensions. Direct interparticle contacts in the gelled sample are more important for heat transfer, as can also be seen in the case of alumina nanoparticle suspensions. Note that despite the formation of chain-like structures in the gelled sample, the chains have no preferential orientation and, therefore, the material can be treated as isotropic as far as thermal conductivity measurements are considered.

We observe more pronounced thermal conductivity enhancement of $3 \%$ for fluid and gel alumina samples than for silica samples even at much lower volume fractions. This could be attributed to the much higher thermal conductivity of pure alumina $\left(33.09 \mathrm{~W} \mathrm{~K}^{-1} \mathrm{~m}^{-1}\right)^{40}$ than pure silica $\left(1.38 \mathrm{~W} \mathrm{~K}^{-1}\right.$ $\left.\mathrm{m}^{-1}\right) .^{41}$

In glass samples the particles are frozen in cages formed by neighboring particles due to the highly expanded electrical double layer. Therefore, we expected to see the influence of solvent layering at the particle surfaces. Surprisingly, we detected significantly decreased heat transfer of the samples in comparison with water. That tendency could be explained in terms of changing water properties, for example, restricted mobility of water molecules in the particles electrical double layer. Water shows a strong decrease of thermal conductivity with lowering temperature and a similar tendency can be expected due to immobilized water. This hypothesis is also supported by the strong decrease of the effect with lowering particle volume fraction, i.e., with the amount of immobilized water molecules.

\section{Conclusions}

To understand the mechanism of heat transfer, we have prepared different states of nanofluids based on the same nanoparticles, i.e., $22 \mathrm{~nm} \mathrm{SiO}_{2}$ and $30 \mathrm{~nm} \mathrm{Al}_{2} \mathrm{O}_{3}$ particles, in aqueous suspension. We investigated the fluid, the gel, and the glass states at equal volume fractions. Rheology was performed to show the behavior of different colloidal states on the macroscopic level. The gelled and glassy silica samples demonstrate a shear-thinning behavior whereas the silica fluid exhibits the typical behavior of a Newtonian fluid with an average particle diameter of $22 \mathrm{~nm}$. Dynamic light scattering was used to inspect the local dynamics of the particles in the glass, the gel, and the fluid colloids. The silica and alumina samples in the gel and glass states demonstrate almost the same behavior of nonergodic colloids as a result of structure formation. In contrast, the fluid samples demonstrate more or less classical Brownian motion of particles. The silica nanofluid is a monodisperse suspension with a hydrodynamic particle radius of $16 \mathrm{~nm}$. The DLS study of alumina nanofluid reveals a polydisperse system with clusters of about $100 \mathrm{~nm}$.

Thermal conductivity of the three states was measured as a function of volume fraction. For the fluid and gel states, thermal conductivity increases almost linearly with concentration. Both the silica and alumina colloids in the gel state exhibit the highest thermal conductivity enhancements, i.e., $18 \%$ enhancement for $\mathrm{SiO}_{2}$ at a volume fraction of $31 \mathrm{vol} \%$ and $10 \%$ enhancement for $\mathrm{Al}_{2} \mathrm{O}_{3}$ at 4.8 vol \%. In contrast, in the glass state thermal conductivity strongly decreases with increasing volume fraction. The drastic difference in thermal conductivity enhancement of the glass $(-25 \%)$ and the gel $(+18 \%)$ is rather surprising and requires deeper investigation.

\section{References and Notes} 27,3 .

(1) Das, S. K.; Choi, S. U. S.; Patel, H. E. Heat Transfer Eng. 2006,

(2) Kang, H. U.; Kim, S. H.; Oh, J. M. Exp. Heat Transfer 2006, 19 , 181.

(3) Maxwell, J. C. Electricity and magnetizm; Clarendon Press: Oxford, U.K., 1873.

(4) Assael, M.; Metaxa, I.; Kakosimos, K.; Constantinou, D. Int. J. Thermophys. 2006, 27, 999.

(5) Hamilton, R.; Crosser, O. K. Ind. Eng. Chem. Fundam. 1962, 1, 187.

(6) Eapen, J.; Li, J.; Yip, S. Phys. Rev. E 2007, 76, 062501.

(7) Prasher, R.; Evans, W.; Meakin, P.; Fish, J.; Phelan, P.; Keblinski, P. Appl. Phys. Lett. 2006, 89, 143119 . 1529.

(8) Prasher, R.; Phelan, P. E.; Bhattacharya, P. Nano Lett. 2006, 6,

(9) Xuan, Y.; Li, Q.; Hu, W. AIChE J. 2003, 49, 1038.

(10) Philip, J.; Shima, P. D.; Raj, B. Appl. Phys. Lett. 2008, 92, 043108.

(11) Wang, B.-X.; Zhou, L.-P.; Peng, X.-F. Int. J. Heat Mass Transfer 2003, 46, 2665.

(12) Huaqing, X.; Jinchang, W.; Tonggeng, X.; Yan, L.; Fei, A.; Qingren, W. J. Appl. Phys. 2002, 91, 4568.

(13) Hwang, Y. J.; Ahn, Y. C.; Shin, H. S.; Lee, C. G.; Kim, G. T.;

Park, H. S.; Lee, J. K. Curr. Appl Phys. 2006, 6, 1068.

(14) Krishnamurthy, S.; Bhattacharya, P.; Phelan, P. E.; Prasher, R. S. Nano Lett. 2006, 6, 419.

(15) Eastman, J. A.; Choi, S. U. S.; Li, S.; Yu, W.; Thompson, L. J. Appl. Phys. Lett. 2001, 78, 718.

(16) Choi, S. U. S.; Zhang, Z. G.; Yu, W.; Lockwood, F. E.; Grulke, E. A. Appl. Phys. Lett. 2001, 79, 2252.

(17) Keblinski, P.; Phillpot, S.; Choi, S.; Eastman, J. Int. J. Heat Mass Transfer 2002, 45, 855 .

(18) Leong, K. C.; Yang, C.; Murshed, S. M. S. J. Nanopart. Res. 2006, $8,245$.

(19) Lee, D. Langmuir 2007, 23, 6011

(20) Jang, S. P.; Choi, S. U. S. Appl. Phys. Lett. 2004, 84, 4316.

(21) Prasher, R.; Bhattacharya, P.; Phelan, P. E. Phys. Rev. Lett. 2005, $94,025901$.

(22) Gharagozloo, P. E.; Eaton, J. K.; Goodson, K. E. Appl. Phys. Lett. 2008, 93, 103110

(23) Peterson, G. P.; Li, C. H. Heat and Mass Transfer in Fluids with Nanoparticle Suspensions. In Advances in Heat Transfer; Harnett, J. P., Irvine, T. F., Eds.; Pergamon Press: New York, 2005; Vol. 39, p 257.

(24) Keblinski, P.; Thomin, J. Phys. Rev. E 2006, 73, 010502.

(25) Evans, W.; Fish, J.; Keblinski, P. Appl. Phys. Lett. 2006, 88, 093116.

(26) Evans, W.; Fish, J.; Keblinski, P. J. Chem. Phys. 2007, 126, 154504.

(27) Patel, H. A.; Garde, S.; Keblinski, P. Nano Lett. 2005, 5, 2225.

(28) Shalkevich, A.; Stradner, A.; Bhat, S. K.; Muller, F.; Schurtenberger, P. Langmuir 2007, 23, 3570.

(29) Urban, C.; Schurtenberger, P. J. Colloid Interface Sci. 1998, 207, 150

(30) KD2Manual; Decagon.

(31) Lu, P. J.; Zaccarelli, E.; Ciulla, F.; Schofield, A. B.; Sciortino, F.; Weitz, D. A. Nature 2008, 453, 499.

(32) Hunter, R. J. Foundations of Colloid Science; Oxford University Press: New York, 2001.

(33) Beck, S.; Hartl, W.; Hempelmann, R. J. Chem. Phys. 1999, 111, 8209.

(34) Zaccarelli, E.; Andreev, S.; Sciortino, F.; Reichman, D. R. Phys. Rev. Lett. 2008, 100, 195701.

(35) Dawson, K. A.; Lawlor, A.; DeGregorio, P.; McCullagh, G. D.; Zaccarelli, E.; Foffi, G.; Tartaglia, P. Faraday Discuss. 2002, 123, 1.

(36) Segre, P. N.; Prasad, V.; Eld, A. B. S.; Weitz, D. A. Phys. Rev. Lett. 2001, 86, 6042.

(37) Kobayashi, M.; Skarba, M.; Galletto, P.; Cakara, D.; Borkovec, M. J. Colloid Interface Sci. 2005, 292, 139.

(38) Gittings, M. R.; Saville, D. A. Colloids Surf. A: Physicochem. Eng. Aspects 1998, 141, 111 .

(39) Chen, G.; Yu, W.; Singh, D.; Cookson, D.; Routbort, J. J. Nanopart. Res. 2008, 10, 1109.

(40) Lide, D. R., Ed. CRC Handbook of Chemistry and Physics, 88th ed.; CRC: Boca Raton, FL, 2007.

(41) Grove, A. S. Physics and Technology of Semiconductor Devices; John Wiley and Sons: New York,1971. 